\title{
INDIVIDUO Y SOCIEDAD EN EL "PROLOGUS" DEL SUPER ETHICA DE ALBERTO MAGNO
}

Francisco Bertelloni ${ }^{*}$

SINTTESE - Alberto Magno foi o primeiro grande comentador de Aristóteles entre os cristãos. Em seu Prólogo à Ética a Nicômaco, defronta-se ele com a divisão da philosophia moralis em individual, econômica e política. Seus antecessores, porém, não faziam uniäo entre os três ramos, enquanto Alberto, usando praticamente os mesmos argumentos e reinterpretando-os a seu modo, consegue recuperar a unidade da ética.
ABSTRACT - Among the Christian philosophers, Albertus Magnus was the first great commentator of Aristotle's work. In his Prologue to the Nichomachean Ethics, he finds the division of the philosophia moralis into three parts, namely individual, economical and political ethics. His predecessors didn't join together the three aspects. Albertus restores the unity.

\section{1 - Introducción}

En trabajos anteriores ${ }^{1}$ he procurado reconstruir algunos elementos teóricos referidos a la concepción de la philosophia moralis, tal como esos elementos están contenidos en los escritos conocidos bajo el nombre común de divisiones philosophiae, surgidos en la primera mitad del siglo XIII en el ámbito de los maestros de artes parisinos. De los elementos presentes en esa concepción que los artistas sostuvieron acerca de la philosophia moralis, dos son particularmente relevantes en relación con el pensamiento que Alberto Magno expone en el Prologus a su tratado Super Ethica. ${ }^{2}$ El primero es la ausencia de vinculos sistemáticos entre las tres partes de la philosophia moralis (ethica, oeconomica y politica) y el consiguiente aislamiento entre esas partes. Precisamente, la causa del aislamiento entre las partes de

- Universidade de Buenos Aires - Argentina

1 Crr. 'De la política como 'scientia legislativa' a lo político 'secundum naturam' (Alberto Magno receptor de la Politica de Aristóteles)", en: Patristica et Mediaevalia XII (1991), pp. 3/34 y "Die Rolle der Natur in den 'Commentarii in Libros Politicorum Aristotelis' des Albertus Magnus, en: Mensch und Natur im Mittelalter, ed. A. Zimmermann, Berlin - New York, 1992, 682-700 (= Miscellanea Mediaevalia, Bd. 21/2), esp. pp. 691 ss.

2 "Alberti Magni Super Ethica. Commentum et Questiones", en: Alberti Magni Opera Omnia, T. XIV, Pars I-II, ed. W. Kübel, Münster 1968, 1972, 1987. Las citas refieren a página y columna de esta edición. 
la philosophia moralis residia en la ausencia de vínculos sistemáticos entre cada una de ellas. El segundo elemento es la insistencia de las divisiones philosophiae en una identificación infundada de una de esas tres partes, "la politica", con la lex positiva. La consecuencia de esa ecuación "politica" = lex fue una concepción exclusivamente jurídica de la "politica" y la consiguiente exclusión de toda posibilidad de instaurar un discurso teórico-especulativo de carácter filosófico acerca de ella.

Desde el punto de vista de la historia del desarrollo de la ética medieval y particularmente desde la perspectiva de las disciplinas que se desprenden de la ética y que comienzan a desarrollarse científicamente a partir del siglo XIII - la politica, por ejemplo - es particularmente relevante comprobar que en el Prologus del Super Ethica, Alberto Magno recibe la concepción que le transmite la tradición de las divisiones philosophiae, la asume con todas la falencias que estaban presentes en esa tradición y luego supera esa concepción intentando dar una respuesta propia y puntual a cada una de esas falencias. De ese modo, al mismo tiempo que Alberto asume la tradición, intenta superarla desde dentro mismo de esa tradición. Así, al problema del aislamiento de cada una de las partes de la philosophia moralis y a la ausencia de vínculos entre esas partes, Alberto responde con una doctrina sistemática que muestra el modo como esas partes se relacionan entre sí superando su aislamiento. Y al problema de la identificación infundada de la politica con la lex positiva, Alberto responde ofreciendo un sólido fundamento de dicha identificación y mostrando el modo como, a través de la lex, la politica se extiende hacia cada una de las otras partes de las philosophia moralis (oeconomica y ethica) relacionándose estrechamente con ellas.

Estas simultáneas asunción y superación por parte de Alberto de los datos transmitidos por la tradición de las divisiones philosophiae constituyen un dato histórico relevante. En efecto, ello muestra la importancia que el Doctor Universalis atribuyó a esa tradición, hasta el punto de considerarla merecedora de constituir, en su Prologus, el punto de partida de sus propias reflexiones sobre la naturaleza de la philosophia moralis. En otros términos, la asunción y la superación de la tradición habla de una suerte de dependencia de Alberto respecto de esa tradición de escritos provenientes del ambiente de los maestros de artes, autores de las divisiones philosophiae.

En este trabajo intentaré insistir sobre esa dependencia de Alberto respecto de esa misma tradición, pero sirviéndome del análisis de otros elementos del Prologus, distintos de los que he considerado hasta ahora. Estos nuevos elementos revelan tambien la importancia que Alberto atribuye a esa tradición de los artistas que le precede en forma inmediata. El tema de mi análisis será, en este caso, la concepción de Alberto acerca de la unidad de la philosophia moralis y el vínculo sistemático que, según Alberto, permite el tránsito desde el carácter unitario de esa philosophia moralis hacia cada una de sus partes, según se trate de la ethica individualis por una parte, o de la ética social - que abarca la oeconomica y la politica - por la otra. Ese vínculo que Alberto reclama y que ha sido olvidado por la tradición que Alberto critica es el vínculo que tipifica la vida ética del hombre como individuo o su vida ética como integrante de la sociedad doméstica o política. 


\section{2 - Alberto asume y supera la tradición}

En el Prologus de su tratado Super Ethica Alberto formula cinco preguntas que apuntan a determinar cientificidad, ${ }^{3}$ unidad, ${ }^{4}$ tema, ${ }^{5}$ fin $^{6}$ y modo ${ }^{7}$ de la disciplina moralis, philosophia moralis o ética general. No se trata de preguntas meramente retóricas. Alberto aspira a que su respuesta a ellas constituya un programa que sirva de fundamento a la nueva construcción de la ética. De estas preguntas analizará la segunda (utrum disciplina moralis sit una) para intentar mostrar, a través de ese análisis, que las fuentes que Alberto utiliza para construir su respuesta son los escritos llamados divisiones philosophiae de la primera mitad del siglo XIII.

Esta respuesta de Alberto constituye un punto de inflexión en el desarrollo de la ética medieval. En efecto, superando la tradición, Alberto traza una linea separatoria entre dos períodos de ese desarrollo. Los argumentos de la tradición, con los que Alberto se confronta en el Prologus, forman parte del primer período. Alberto percibe una falencia en el tratamiento que hace la tradición del problema de la unidad de la ética y se propone superar esa falencia.

Este trabajo se plantea un doble objetivo. En primer lugar debe mostrar que aunque Alberto critica los argumentos de la tradición, sin embargo su crítica se apoya en argumentos que encuentra en la misma tradición que critica. En ambos casos se trata de la tradición de las divisiones philosophiae emanadas del ámbito de los artistas de la primera mitad del siglo XIII, es decir del período inmediatamente anterior a la redacción del Super Ethica (1248-1252). En segundo lugar debe mostrar en qué medida este proceder de Alberto facilita el tránsito hacia el segundo período del desarrollo de la ética medieval.

Tanto en la tradición, cuyos argumentos contra la unidad de la philosophia moralis Alberto menciona y de inmediato critica, como en la argumentación que Alberto utiliza para superar esa tradición, aparecen principios y argumentos cuyo origen Alberto o bien esconde o bien determina incorrectamente. Se trata de argumentos que ya fueron utilizados con frecuencia en el ambiente de los artistas de la primera mitad del siglo. De ese modo, Alberto se instala dentro de la tradición para criticar esa tradición desde dentro mismo de ella. Ello posibilita el tránsito desde el primer hacia el segundo período del desarrollo de la ética medieval.

El § 3 muestra, por una parte, en qué consiste, según Alberto, la falta de sistematicidad interna y de unidad de la disciplina moralis en el tratamiento que de ella hace la tradición; por la otra muestra que dicha ausencia de sistematicidad y de unidad no provienen del comentador griego Eustratio - como lo sostiene Alberto sino de algunos textos de la tradición de las divisiones philosophiae de la primera mitad del siglo. El § 4 presenta, en primer lugar, los recursos teóricos a que apela Alberto para superar las falencias señaladas en § 3 , y por la otra, que los argumentos utilizados por Alberto para superar la tradición ya se encontraban entre los maestros de artes de la primera mitad del siglo, es decir, en la misma tradición que

3 "utrum de moribus possit esse scientia" (crr. ibid., 1b).

4 "utrum disciplina moralis sit una" (crr. ibid., 2a).

5 "Circa tertium, scilicet subiectum huius scientiae" (cir. ibid., 3a).

6 "quarto quaeritur, quis sit finis huius scientiae" (cfr. ibid., 4a).

7 "quinto quaeritur de modo" (cfr. ibid., 4 b). 
Alberto critica. El § 5 evalúa el procedimiento de Alberto en el Prologus del Super Ethica y el significado de este procedimiento para el desarrollo de la historia de las ideas medievales.

\section{3 - Falencias en el tratamiento que hace la tradición del problema de la unidad de la ética}

Alberto se confronta con la tradición cuando trata el problema utrim disciplina moralis sit una. Somete el problema a un examen cuidadoso utilizando cuatro argumentos que - según Alberto - ponen en duda la unidad de la disciplina moralis. Alberto insiste especialmente sobre el cuarto argumento - que atribuye al comentador griego Eustratio - y sostiene que de ese argumento ${ }^{8}$ resulta una falta de unidad entre las distintas partes de la disciplina moralis, es decir entre ethica, oeconomica y politica. Por ello, no se trataría de una ciencia unitaria, sino de tres ciencias diferentes entre si: "[...] ergo non videtur, quod istae tres conveniant sub una scientia". 9

Dentro del contexto de la estructura general de la pregunta por la unidad de la disciplina moralis, Alberto presenta el argumento que atribuye a Eustratio como la objeción principal contra la unidad de la ética. Asi, cuando contra ese argumento Alberto defiende la unidad de la ética, presenta su propia solutio no solamente como solución general a toda la quaestio, sino como respuesta dirigida directamente a la objeción que atribuye a Eustratio: "Ad quartum solutio patet". ${ }^{10}$ De ese modo Alberto se confronta directamente con el argumento atribuido al comentador griego.

Ese rol central que Alberto atribuye al argumento que atribuye a Eustratio exige someter ese argumento a un análisis articulado en dos momentos: (a) el primero constituye una comparación del argumento atribuído por Alberto a Eustratio con lo que éste realmente afirmó acerca del problema de la unidad de la disciplina moralis; (b) el segundo es un análisis de las ideas que Eustratio sostuvo acerca de la unidad de la disciplina moralis en su comentario al primer libro de la Etica de Aristóteles.

(a) De la comparación resulta, primero, que Eustratio no se refiere a diferentes tratados en los que ethica, oeconomica y politica son tratadas diferentemente y en forma separada una de las otras; ${ }^{11}$ segundo, Eustratio tampoco habla de una diffe-

8 "Praetera, in commento dicitur, quod tres tradidit scientias de moribus hominum, scilicet moralem, oeconomicam et politicam vel legis positivam, et sicut istae tres diversificantur in tractatibus, sic differunt in finibus, quia moralis finis est felicitas, oeconomicae divitiae, civilis rei publicae tranquillitas; differunt etiam in materia, quia primae humanum bonum, secundae domus, tertiae civitas" (cfr. Super Ethica, 2b).

Cfr. ibid.

10 Cfr. Ibid., 3a.

i1 En el Prologus de su Comentario Eustratio plantea cuatro preguntas: "Primo igitur dicendum quid differunt ad invicem tres species practicae, deinde quae est unaquaeque harum, et tertio quae est ex unaquaque harum hominibus adveniens utilitas" (cfr. H. P. F. Mercken, The Greek Commentaries on the Nicomachean Ethics in the Latin Translation of Robert Grosseteste, Bishop of Lincoln $\Leftrightarrow$ 1253, vol. I "Corpus Latinum Commentariorum in Aristotelem Graeconum", VI, 1, Leyden 1973, 1, 1416). A la primera pregunta (quid differunt [...] tres species practicae) Eustratio responde refiriéndose a los subiecta, sin mencionar los tratados: "Differunt igitur ad invicem ethica, oeconomica è politica (id est moralis, dispensativa et civilis) primo quidem secundum subiecta [...]" (cfr. ibid., 1, 18-19). 
rentia secundum materiam, sino secundum subiecta; ${ }^{12}$ en tercer lugar el comentador griego tampoco sostiene una diferencia in finibus. Eustratio sostiene más bien una separación entre las distintas partes de la philosophia practica; esa separación no depende tanto de los fines como de la utilitas de cada parte para el hombre. ${ }^{13} \mathrm{~A}$ partir de ese momento, las respuestas de Eustratio a las preguntas quae est unaquaeque harum y a la pregunta por la utilitas ${ }^{14}$ son menos satisfactorias que su respuesta a la pregunta por el subiectum de cada una de las partes. En lugar de ello se dedica a una presentación general de cada una de esas partes en la cual sus definiciones (quae est unaquaeque harum) y su utilidad pasan a segundo plano. En esta presentación Eustratio no logra definir con claridad las diferencias entre ambas. $^{15}$

(b) Independientemente del hecho de que Alberto atribuye a Eustratio doctrinas que éste no sostiene, debemos verificar ahora si en su tratamiento del problema de la unidad de la philosophia practica Eustratio sostiene doctrinas que ponen en peligro dicha unidad.

Aunque en el Prologus a su comentario Eustratio muestra una tendencia a considerar cuantitativamente las diferencias entre las distintas partes de la philosophia practica, ${ }^{16}$ el comentador griego supera rápidamente esa tendencia en el cuerpo de su comentario cuando atribuye a la politica una primacía lógico-ontoló-

12 Cfr. supra, nota 10 in fine. Eustratius no menciona la materia, sino el subiectum, pero no el de la philosophia practica, sino que se refiere muy precisamente y en forma exclusiva a los subiecta de cada una de las partes de la philosophia practica y define asi el subiectum de cada parte: "[...] ethicae quidem subiectum est secundum unum hominem melioratio" (cfr. ibid., 2, 20-1); "Oeconomicae autem domus et inhabitantes subiecta et quaecumque his conveniunt et conferunt ad consistentiam simul et cum directione cohabitationem [...]" (cfr. ibid., 2, 40-2); "Politicae autem civitas subiectum et qui in ipsa communicant habitatione* (cfr. ibid., 3, 53-4).

13 V. supra, nota 10 , in principio.

14 Cfr. ibid.

15 Eustratio se ocupa de la utilitas de la ethica y se refiere en particular a la función de la ratio, pero olvida dar una definición de la ethica: "[...] ut 'homo' et bonus et optimus fiat sequens ea quae tradita sunt in morali negotio, prudenter vivens et propriam rationem habens, irae et concupiscentiae dominans et mensuram motibus earum imponens et nequaquam concedens eis ut contingit ferr, ut in tali quis habitu constituatur, ut de omni quod operatur paratus sit rationem reddere rectam secundum quam perfectum est quod ab eo est operatum [...]" (cfr. como nota 7, 2, 21-27). En lo que concieme a la oeconomica, tambien en este caso Eustratio se limita a su utilitas ("[...] ut adipiscatur unusquisque ea quibus indiget secundum analogiam et nullus privetur aliquo conveniente secundum mensuram moris vel ordinis vel habitus vel operationis vel subexistentis naturalis aptitudinis [...]", ibid. 2, 42-45). Su caracterización de la politica es mas detallada, ya que no se ocupa de su utilitas, sino que ofrece una definición de la politica: "Est autem politica quidem moralis species philosophiae practicae [...] circa consistentiam et concordiam civitatis et conversantium in civitate occupationem demonstrans" (ibid., 4, 92-94). Además de ello caracteriza la politica comparándola con la oeconomica y menciona sus partes.

En su caracterización de las distintas partes de la philosophia practica Eustravio parte del subiectum de la ethica, al que define secundum unum hominem melioratio (cfr. ibid., 2, 20-1). Despues de haber definido la ethica sobre la base del individuo, es decir cuantitativamente, se refiere a la domus y a sus inhabitantes como subiecta de la oeconomica (cfr. ibid., 2, 40). Finalmente insiste nuevamente en una definición de la politica de acuerdo al número de súbditos, ya que describe su subiectum die civitas - como ex multis domibus constituitur (cf. ibid., 3, 53-4). Estas caracterizaciones de Eustratio ponen en peligro la unidad de la disciplina moralis porque descuidan las diferencias esenciales entre ethica, oeconomica y politica y de ese modo cierran el camino a la posibilidad de encontrar un fundamento filosófico-sistemático común a todas las partes de la philosophia moralis. 
gica sobre las otras partes de la philosophia practica. ${ }^{17}$ Esta primacía muestra que tambien para Eustratio la politica está por encima de las otras partes de la philosophia practica en la medida en que los fines de la ethica y la oeconomica están subordinados a los fines de la politica. Así Eustratio conserva el rol unificador de la politica respecto de toda la philosophia practica permaneciendo fiel a la doctrina aristotélica de las diferencias esenciales entre ethica, oeconomica y politica y de la unidad de toda la philosophia moralis. ${ }^{18}$

Resumiendo las conclusiones de (a) y (b) tenemos, en primer lugar, que Eustratio no ha sostenido las doctrinas que le atribuye Alberto y en segundo lugar que Eustratio no pone en peligro la unidad de la philosophia practica. Ello sugiere la necesidad de plantear dos preguntas: ¿porqué Alberto atribuye dichas doctrinas al comentador griego? y ¿de dónde provienen esas doctrinas si ellas no provienen de Eustratio?

En lo que concierne a la primera pregunta, no es fácil explicar porqué Alberto omite mencionar la tradición a la que se está refiriendo. Al respecto solo puedo hacer referencia a otros pasajes del Prologus en los que procede de modo similar. En un trabajo anterior he podido comprobar que Alberto tambien se refiere a explícitamente a Eustratio mientras que, en rigor, omite mencionar su fuente real, es decir la tradición de los artistas de la primera mitad del siglo XIII. ${ }^{19}$

La pregunta por el origen de las doctrinas que Alberto utiliza sin mencionar es más fácil de responder. Ellas no provienen de Eustratio, sino con absoluta certeza de la tradición de las divisiones philosophiae de la primera mitad del siglo XIII. Alberto verifica en esa tradición una descomposición de la philosophia moralis en sus partes, de modo tal que éstas aparecen dispersas y totalmente aisladas entre sí. Solo a partir de esta tradición Alberto puede concluir: "ergo non videtur, quod istae tres conveniant sub una scientia". Debe tenerse en cuenta que, muy probablemente, esta tradición de los artistas se retrotrae a las tradiciones latinas inauguradas por Boecio y Casiodoro y continuadas por Isidoro de Sevilla y Hugo de San Victor, en cuyas presentaciones de la philosophia practica sus partes aparecen totalmente carentes de relaciones sistemáticas y, consecuentemente, totalmente aisladas entre si. ${ }^{20}$

17 "Propositum est hic ostendere politicam esse omnibus supereminentem artibus et principalem et dominam omnium quae in humanis communicationibus sunt adinventionum [...]" (cfr. ibid., 16, 90-2). "Subiacent enim ipsi 'politicae' oeconomica et ethica [...]" (cfr ibid., 26, 68-9).

18 "Puesto que la politica se sirve de las otras ciencias prácticas para sus propios fines [...] el fin de la politica abarca los fines de las esas otras ciencias [...] " (cfr. Eth. Nic., I, 2, 1094b, 4-6). "Se equivoca el que piense que entre el jefe político, el rey, el jefe de familia y el señor de esclavos no existe ninguna diferencia esencial* (crf. Politica, I, 1, 1252a, 6-10). Cfr. F. Bertelloni, "Die Rolle der Natur [...]" (como nota 1), esp. pp. 691 ss.

20 "Practicae vero philosophiae [...]triplex est divisio; est enim prima quae sui curam gerens cunctis sese erigit, exomat augetque virtutibus, nihil in vita admittens quo non gaudeat, nihil faciens paenitendum; secunda vero est quae rei publicae curam suspiciens cunctorum saluti suae providentiae sollertia et iustitiae libra et fortitudinis stabilitate et temperantiae patientia medetur; tertia vero, quae familiaris rei officium mediocri componens dispositione distribuit" (cfr. Boethius, In Isagogen Porphyni commenta, ed. G. Schepss / S. Brand, CSEL 48, Vindobonae-Lipsiae, 1906, 9). "Moralis dicitur, per quam mos vivendi honestus appetitur, et instituta ad virtutem tendentia praeparantur. Dispensativa dicitur, domesticarum rerum sapienter ordo dispositus. Civilis dicitur, per quam totius ci- 
Si se procura reconstruir la situación de la philosophia practica en las divisiones philosophiae de la primera mitad del siglo XIII, se deben tener en cuenta las observaciones de G. Wieland sobre el tema, quien ha mostrado que en general, los artistas han insistido en la articulación tripartita de la philosophia practica en ethica, oeconomica y politica, sin lograr definir con precisión los vinculos sistemáticos entre esas partes. ${ }^{21}$ A ello debería agregarse que, en esos textos, la ausencia de sistematicidad se agudiza por tres motivos: primero, porque las divisiones philosophiae tienden a considerar las diferencias entre las partes de la philosophia practica de modo puramente cuantitativo; segundo, porque como consecuencia de ello, no atienden a las características ontológicas de cada parte, descuidando asi las diferencias esenciales entre ellas; ${ }^{22}$ y tercero, porque ellos no profundizan los vínculos que comprometen al individuo ni considerado desde el punto de vista de la ética individual (ethica) ni desde el de la ética social (oeconomica y politica).

Así las divisiones philosophiae en general muestran una concepción de la philosophia moralis según la cual ésta se disuelve en sus partes y pierde su unidad. Como ejemplo de esta concepción encontramos diversos textos que presentan las mismas características que Alberto verifica en los argumentos que atribuye a Eustratio y que, según Alberto, actúan como causa de la pérdida de unidad de la philosophia moralis. En síntesis esas características son: (a) articulación de la philosophia moralis en tres partes; (b) identificación de cada parte con un determinado tratado; (c) aislamiento y cerramiento de cada parte en sí misma, sin relación con las otras; (d) tendencia a la consideración de las diferencias cuantitativas entre las partes que solo permiten un tránsito cuantitativo hacia las otras partes; (e) identificación de la politica con las leges y los decreta, i. e. con la lex positiva y (f) ausencia de toda referencia a la esencial discontinuidad entre la ética individual (ethica) por una parte y la ética social (oeconomica y politica) por la otra. ${ }^{23}$

vitatis administratur utilitas" (cfr. Cassiodorus, De artibus et disciplinis liberalium litteratum, III, en: Migne, PL, 70, 1169). "Moralis dicitur, per quam vivendi mos honestus appetitur et instituta ad virtutem tendentia praeparantur. Dispensativa dicitur, cum domesticarum rerum sapienter ordo disponitur. Civilis dicitur per quam totius civitatis utilitas administratur" (cfr. Isidoro de Sevilla, Etymologiarum [...] Libri XX, II, 24. 16, en: Migne, PL, 82, 142). "Moralis dicitur, per quam mos vivendi honestus expetitur vel acquiritur, et instituta ad virtutem tendentia praeparantur. Dispensativa dicitur, cum domesticarum rerum sapienter ordo disponitur. Civilis dicitur, per quam totius civitatis utilitas administratur" (cfr. Hugo de San Victor, Didascalion, II, xix, en: Migne, PL, 176, 760-1).

21 Crr. G. Wieland, Ethica-Scientia practica. Die Anfänge der philosophischen Ethik im 13. Jahrhundert, Münster, 1981 (Beiträge zur Geschichte der Philosophie des Mittelalters, NF. XXI), pp. 95 ssq.

22 A esas dos caracteristicas de las divisiones philosophiae me refiero en "Die Rolle der Natur [...]" (como nota 1), pp. 685-90.

23 Ejemplos de dicha concepción sdon los siguientes textos: Philosophia de Oliverus Brito: "Practica 'philosophia' dividitur in monosticam, yconomicam et politicam. Monostica est quae ordinat hominem ad regimen sui, et dicitur a monos, quod est unus, et ycos, quod est scientia, quasi scientia unius [...] Et hec datur ab Aristotile in Ethicis. Yconomica est que docet propriam familiam ordinare, unde yconomos grece, dispensator latine; vel dicitur ab Yconomo, huius scientie primitur inventore. Et hec a Tullio libro suo De Officiis enodatur. Politica nominatur que docet regere populos et civitates, unde dicitur a polis, quod est civitas vel pluralitas. Et hec traditur in legibus et decretis" (MS Oxford, Bodleian Library, Corpus Christi College, 283 fol. 151 ra-b; cfr. R. A. Gauthier, "Amoul de Provence et la doctrine de la 'Fronesis', vertu mystique supreme" en: Revue du moyen Age latin 19 (1963), p. 143); Divisio scientiarum Amulfi Provincialis: "Est autem triplex modus vivendi et conversandi. Unus cum subditis, militibus et civibus, qui consistit in regimine populi, regionis vel civitatis, et de illo est una pars moralis que dicitur politica, dicta a polis, quod est pluralitas, et ycos, scientia, 
Así, la concepción de la philosophia moralis sostenida en el ámbito de los artistas cierra toda posibilidad de encontrar los vínculos sistemáticos que relacionan entre sí a las tres partes de la philosophia practica. Como consecuencia de esta carencia de sistematicidad y en contraposición a la concepción aristotélica de la división tripartita de la philosophia moralis, las divisiones philosophiae no solamente tratan cada una de las partes aisladamente, sino que en ellas se echa de menos una distinción filosófica entre las peculiariedades de la ética del individuo y las de la ética social (oeconomica y politica). La tradición sobre la que Alberto dirige sus críticas en el Prologus no ha elaborado ni la especificidad de la ética individual i. e. el hombre en relación consigo mismo - ni la de la ética en relación con otros hombres en el ámbito de la domus o en el de la civitas.

\section{4 - La fundamentación de Alberto de la "philosophia moralis": "homo dupliciter potest considerari"}

En su respuesta Alberto procura superar esa tradición. Su confrontación con ella puede ser considerada, desde un punto de vista sistemático-doctrinal, como un intento de superación de las falencias de la tradición a través de la formulación filosófica de las diferencias entre la ética individual y la ética social. Desde el punto de vista de la historia de las ideas, el proceder de Alberto se caracteriza fundamentalmente por un hecho paradojal, ya que utiliza argumentos que habían sido difundidos por la misma tradición que Alberto procura superar.

Alberto articula su respuesta en dos momentos. Por una parte, (a) procura superar la ruptura de la unidad de la philosophia moralis como respuesta a las consecuencias de una doctrina que - como sostiene Alberto - fue sostenida por Eustratio. Por la otra, (b) se opone a un fallido intento de fundamentación de la unidad de la philosophia moralis que Alberto tambien atribuye a Eustratio.

(a) Según la doctrina atribuída por Alberto a Eustratio, ethica, oeconomica y politica se diferencian tan radicalmente entre si in tractatibus, in finibus e in mate-

quasi scientia de regimine plurium. Et hanc dicunt quidam haberi per leges et decreta; alii a Tullio traditam esse in quibusdam libris qui non multum a nobis habentur in usu. Alius est modus conversandi cum propria familia, de quo est alia pars moralis, que dicitur yconomica, dicta, ut exponunt quidam, a yconomos, quod est dispensare et ycos, scientia, quasi scientia dispensandi et regendi propriam familiam. Et ista dicitur tradi a Tullio in quodam libro de regimine familie. Tertius est modus vivendi et conversandi secum propriam animam gubernando, de quo est pars moralis, que dicitur monostica, dicta a monos, quod est unum, et ycos, scientia, quasi scientia de regimine unius, scilicet sui ipsius" (cfr. C. Lafleur, Quatre introductions à la Philosophie au XIIe siècle, Montrèal Panis 1988, p. 333). Véase además: Anonymus Sicut dicit Isaac (MS München Clm., 14460, fol. 168 r; cfr. M. Grabmann, "Die logischen Schriften des Nikolaus von Paris und ihre Stellung in der aristotelischen Bewegung des XIII. Jahrhunderts", en: Mittelalterliches Geistesleben, T. I, München, 1926, p. 245. Según Grabmann, este texto proviene de Nicolás de Paris, tesis que Lafleur (cfr. Quatre introductions [...], p. 391, nota 12) rechaza; MS Bruges, Bib. de la Ville 496, fol. 80 ra (cfr. Gauthier, loc. cit. p. 141); Pseudo Adam de Bocfeld (Cod. G. 4355 de la Biblioteca Nazionale, Florenz, fol. 91 v; cfr. M. Grabmann, "Die Aristoteleskommentatoren Adam von Bocfeld und Adam von Bouchermefort", en: Mittelalterliches Geistesleben, T. II, München, 1936, p. 155; se trata del comentario al Liber de Causis atribuido por M. Grabmann a Adam de Bouchermefort; véase sobre esta atribución Gauthier. loc. cit., p. 141, nota 32); Henricus Brito (MS Oxford, Bodleian Library, Corpus Christi College 283, fol. 148 va; cfr. Gauthier, loc. cit., p. 144, nota 38); Petrus de Hibernia, Comentario a la Isagoge (cfr. MS Vat. Lat. 5988, fol. 64 rb) y idem., en: MS Vat. Lat. 5988, fol. 82 ra. 
ria, que tanto las posibilidades de su unidad como de mutuas relaciones entre ellas parecen totalmente excluidas. En § 3 hemos presentado ya el origen y las consecuencias teóricas de esta doctrina. Contra esa desarticulación de la philosophia moralis Alberto afirma la unidad de su objeto (subiectum), mientras que sus partes sí pueden ser múltiples (plures). ${ }^{24}$ Además de ello, Alberto asegura, dentro de la disciplina moralis, la existencia de relaciones mutuas entre las partes; lo hace recurriendo a la distinción entre scientia docens y scientia utens: mientras la ethica individual, como scientia docens, suministra principios para la práctica de las costumbres (exercitium morum), la oeconomica y la politica como scientia utens se apoyan en esos principios y apuntan a la aplicación de esos principios. ${ }^{25}$

(b) De inmediato Alberto se dedica al análisis de la fundamentación de la unidad de la disciplina moralis que atribuye a Eustratio. Alberto considera que dicha fundamentación es incorrecta. En 2 ya he señalado que Alberto no solamente impugna por insuficientes los argumentos de la tradición (Eustratio) cuando ésta rechaza la unidad de la disciplina moralis; además de ello Alberto verifica que, a pesar de dicha insuficiencia, esa tradición insiste en buscar un argumento - que Alberto tambien considerará insuficiente - para fundamentar la unidad de la disciplina moralis: "Sed Commentator Graecus aliter vult, quod istae partes uniantur in una ratione subiecti, quia quaelibet earum considerat mores hominis". ${ }^{26}$ En este texto Alberto atribuye nuevamente a Eustratio el intento de fundamentar la unidad de la ética. ${ }^{27}$ Pero tambien en este caso la tradición ha fracasado en la elección de los argumentos correctos, pues ella refiere su fundamentación nada más que a los mores hominis.

Alberto actúa de modo muy consecuente cuando se trata de llamar la atención sobre los errores de la tradición. En un primer momento refuta los argumentos que esgrime la tradición para negar la unidad de la disciplina moralis. ${ }^{28} \mathrm{Y}$ ahora vuelve a refutar los argumentos de esa tradición cuando ésta intenta fundamentar la unidad de la disciplina moralis a cualquier precio, es decir, sobre bases que, nuevamente, Alberto considera falsas. Alberto quiere mostrar que los mores hominis no son suficientes para fundamentar la unidad de la ética. Alberto afirma que ésta se

24 "Dicendum, quod haec est scientia unius generis subiecti, sub qua continentur plures partes sicut sub naturali" (cfr. Super Ethica, 2b)

25

"Tres autem sunt partes huius secundum commentatorem arabem sic differentes, quod in morali traduntur principia moralium [...], in aliis autem duabus traditur exercitium morum secundum usum illorum principionum in specialibus casibus [...]. Sic ergo hic traduntur principia, quibus illae utuntur; quarum una docet, qualiter convivendum sit domesticis, altera, qualiter convivendum sit civibus. Et sic planum est, quod reducuntur ad unam scientiam communem, ex quo ex eisdem principis procedunt (cfr ibid., 2b-3a). Un análisis detallado de este pasaje he realizado en: "Die Rolle der Natur [...]", (como nota 1), pp. $692 \mathrm{ss.}$ Cfr. Super Ethica, $3 \mathrm{a}$.

27 Aunque la edición critica ("ed. Heylbut p. 1 v. 25 sqq.; transl. Gross. f. 1 ra"; cfr Super Ethica, 3, in fine) y A. Pelzer ("Le cours inédit d'Albert le Grand sur la Morale à Nicomaque recueilli et rédigé par $\mathrm{S}$. Thomas d'Aquin" en: id., Études d'Histoire Littéraire sur la Scolastique Médiévale, ed. A. Pattin y E. Van de Vyver, Louvain-Paris, 1964, p. 305, nota 19 y p. 308, nota 22) se refieren al Texto del comentario de Eustratio como fuente de este pasaje, no encuentro en el comentario de Eustratio ningún intento de fundamentar la unidad de las partes de la philosophia practica "in una ratione subiecti".

28 Véase supra, $\$ 3$. 
puede considerar desde dos puntos de vista, según que el hombre sea considerado en relación consigo mismo o en relación con otros hombres: "Sed homo dupliciter potest considerari: vel secundum se vel in comparatione ad alterum, et in comparatione ad alterum dupliciter: vel ad domesticos coniunctos vel ad omnes communiter qui sunt sub eadem civitate vel regno vel gente".$^{29}$ Naturalmente, esta nueva división de la disciplina moralis implica que cada una de sus partes debe regirse por principios diferentes y que esos principios serán específicos para cada parte: "nec omnes per eadem principia regulantur in regimine domus et sui ipsius et civitatis, sed per diversa, sicut patet".$^{30}$ Ello significa el fin de la indiferenciación entre las partes y el comienzo de la búsqueda de la unidad de esas partes a través de la búsqueda de los elementos comunes de esos principios diferentes.

En § 3 hemos mostrado que Alberto en realidad no rechazaba los argumentos de Eustratio, sino que esos argumentos que Alberto atribuye al comentador griego provenían del medio intelectual de los artistas. Ahora debemos mostrar que Alberto lleva a cabo su división de la disciplina moralis en ética del individuo (secundum se) y ética social (in comparatione ad alterum) tomando argumentos que tambien provienen de esa misma tradición artista. En efecto, no todos los textos provenientes del medio intelectual de los artistas han considerado asistemáticamente la estructura interna de la philosophia practica convirtiéndola en una simple suma de tres ciencias diferentes. En particular dos textos relevantes de esa tradición han propuesto una estructura bipartita de la philosophia moralis que especifica principios propios para cada una de ellas.

El primero de ellos es el plan de estudios descubierto por M. Grabmann en 1927 en el Archivo de la Corona de Aragón de Barcelona, editado recientemente por C. Lafleur. ${ }^{31}$ La concepción sobre la ética del anónimo autor de este escrito contempla, por una parte, la dimensión individual de la ética (monastica), cuyo principio o vínculo propio ( = anima vivit ut in se ipsa) recae sobre el mismo individuo y permanece en él. Además, el autor distingue claramente la dimensión social de la ética, que a su vez se subdivide en ypotica y politica. El principio o vínculo propio de esta dimensión social es la vita animae in bono aliorum, es decir la relación con otros hombres. Asi, en este texto, ypotica y politica son consideradas disciplinas o ramificaciones de un mismo principio, cualitativamente diferente del principio propio de la ética individual. ${ }^{32}$

Cfr. Super Ethica, 3a.

Cr. ibid.

Una interpretación del significado de este texto para la ética y la politica medievales ofrezco en mi trabajo sobre "Politologische Ansichten bei den Artisten um 1230/ 1240. Zur Deutung des anonymen Pariser Studienplans Hs. Ripoll 109", en: Theologie und Philasophie, 69 (1994), pp. 34/73.

"Hoc habito, de secunda specie philosophie, scilicet de morali, cuius principium est voluntas 'videndum est'. Hec autem scientia est ut boni fiamus, ut in secundo Ethicorum. Dividitur autem hec scientia secundum multiplicem vitam anime in bono. Dicit autem Augustinus quod anima uno modo totaliter vivit in Deo; et secundum hoc est theologia que tradita solum a spiritu sancto. Et hec maxime convenit anime secundum vitam contemplativam, que vita maxime consistit intellectuali. De qua dicitur in secundo Ethicorum quod virtus multum habet ex doctrina, quia meliores contemplativi sunt ut frequentius qui magis intelligunt de divinis. Anima iterum vivit in bono alionum, et hoc dupliciter. Uno modo in regendo sibi subditos cogitando de bono eorum inquantum potest et ut debet. Et secundum hoc est Liber de vera iustitia vel Liber de officiis - quod idem est - quem fecit Tullius. 
La misma concepción se encuentra en el texto anónimo conocido como Ut ait Tullius. En este escrito, cada una de las partes de la philosophia practica está vinculada con las otras por el hecho de que todas ellas son referidas al principio unificador de toda la scientia practica, es decir al principio común que el texto llama regimen. Pero este regimen se diversifica, a su vez, según se trate de la ética individual (= regimen ipsius) o de la social (= regimen alterius). Así, en este texto, la dimensión social de la filosofía práctica en sus dos disciplinas (yconomica y politica) es presentada como una ramificación de un único principio (regimen alterius) y tratada aún más detallada y cuidadosamente que en el plan de estudios de Barcelona. $^{33}$

Ambos textos son, en lo esencial, similares. En efecto, por una parte la yconomica en el texto Ut ait Tullius y la ypotica en el plan de estudios de Barcelona, y por la otra la politica en ambos textos, son consideradas como ramificaciones del principio regimen alterius en el texto Ut ait Tullius y del principio vita animae in bono aliorum en el plan de estudios de Barcelona. En ambos textos, la dependencia de ypotica/yconomica y de la politica respecto de un principio único que se diferencia cualitativamente del principio de la ética individual (regimen sui en el Ut ait Tullius y vita animae in se ipsa en el plan de estudios) muestra con claridad el propósito de los respectivos autores: primero, separar la dimensión social de la filosofía práctica de su dimensión individual; segundo, fundamentar esa separación en principios diferentes según se trate de la ética individual o social.

Así, en estos dos textos, la tripartición asistemática de la filosofía práctica, tal como esa tripartición aparece en la mayoría de las divisiones philosophiae, es reemplazada por una bipartición sistemática. Mientras que la tripartición presenta-

Et hec scientia ypotica appellatur ab ypos, quod est 'sub', quasi scientia de subditis. Item, anima vivit in bono omnium communiter secundum legem communem, et secundum hoc est scientia que traditur in legibus et decretis. Que politica vocatur a polis, quod est 'civitas', eo quod est de iure et defensione iuris eorum que sunt in civitatibus constituta. Quarto modo anima vivit ut in se ipsa bene regendo potentias sensibiles. Hec vita proprie consistit in virtute consuetudinali, de qua dicitur in secundo Ethicorum quod nomen accipit ab assuetudine. Hec enim virtus maxime acquiritur per assuetudinem bonarum operationum. Et secundum hoc est scientia libri Ethicorum. Est enim ethis grece 'habitus' latine. Et hec scientia alio nomine monostica a monos, quod est 'unum', quia anima secundum hanc virtutem de se proprie cogitat vel de sui bono" (cfr. C. Lafleur, avec la collaboration de Joanne Carrier, Le "Guide de l'Atudiant" d'un maitre anonyme de la Faculté des Arts de Paris au $X \mathrm{XI}^{\mathrm{e}}$ siècle. Adition critique provisoire du ms. Barcelona, Arxiù de la Corona d'Aragó, Ripoll 109, ff. 134 ra-158 va, Faculté de Philosophie, Université Laval, Québec, 1992, pp. 53 ss.).

"Practica vero scientia dividitur in monosticam, yconomicam et politicam, et hec divisio sic sumitur. Practica vero est scientia que ordinat hominem ad regimen ipsius vel alterius. Aut ergo ordinat hominem ad regimen sui tantum et sic est monostica, et dicitur a monos, quod est unum, et ycos, quod est custodia, quasi unius custodia. Aut ordinat hominem ad regimen alterius, et hoc dupliciter, quia aut ad regimen alterius sibi subditi tantum, vel subditi et propinqui. Si subditi tantum, sic est yconomica, que docet qualiter homo sue familie debeat disponere, et dicitur yconomica ab ycos, quod est custodia, vel ab ycos quod est domus, et custos, custodis; tractat enim de custodia domus; vel dicitur ab Yconomo inventore. Si vero ordinet hominem ad regimen sibi subditi et propinqui, sic est politica, per quam homo scit regere civitatem; et dicitur politica a polis, quod est pluralitas vel civitas, et custos, custodis, quasi plurium vel civitatis custos. Practica traditur ab Aristotile in Ethicis, secunda a Tullio in libro De officiis, tertia in legibus et decretis" (cfr. ms. Paris, Bibliotheque Nationale, Nouv. Acq. Lat., 1374, fol. 12 va; cito apud Gauthier, ut supra, nota 22, pp. 142 ss.).

Vide supra, nota 23. 
ba a cada una de las partes de la filosofía práctica sin fundamentarlas en principios específicos, la bipartición recurre a un principio específico para la ética individual y a otro distinto para la ética social. Así, la bipartición logra bloquear el tránsito inmediato - típico de la tripartición - desde la dimensión práctica de la divisio philosophiae (philosophia moralis en el plan de estudios, scientia practica en el Ut ait Tullius) hacia la yconomica/ ypotica y hacia la politica respectivamente. Este bloqueo tiene lugar gracias al recurso a un principio que actúa como mediación entre la filosofía práctica por una parte y la oeconomica y la politica por la otra. El paso desde la filosofia práctica hacia cada una de esas dos partes se produce a través un principio que tipifica la dimensión social de la filosofia práctica.

\begin{tabular}{|c|c|c|c|}
\hline \multirow{4}{*}{} & & \multicolumn{2}{|c|}{ secundum se } \\
\cline { 3 - 4 } 葛 & homo dupliciter & & ad domesticos coniunctos \\
\cline { 3 - 4 } & potest considerari & $\begin{array}{c}\text { in } \\
\text { comparatione } \\
\text { ad alterum }\end{array}$ & $\begin{array}{c}\text { ad omnes communiter qui } \\
\text { sunt sub eadem civitate }\end{array}$ \\
\hline
\end{tabular}

\begin{tabular}{|c|c|c|c|}
\hline \multirow{3}{*}{ 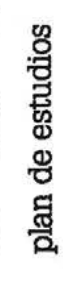 } & \multirow{2}{*}{$\begin{array}{l}\text { philosophia } \\
\text { moralis }\end{array}$} & \multicolumn{2}{|c|}{ anima vivit ut in se ipsa (monastica) } \\
\hline & & & $\begin{array}{l}\text { in regendo sibi subditos } \\
\text { (ypotica) }\end{array}$ \\
\hline & & bono aliorum & $\begin{array}{l}\text { in bono omnium } \\
\text { communiter (politica) }\end{array}$ \\
\hline
\end{tabular}

\begin{tabular}{|c|c|c|c|}
\hline \multirow{3}{*}{ 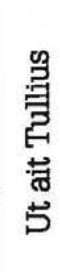 } & \multirow{4}{*}{ scientia practica } & \multicolumn{2}{|c|}{ regimen sui (monastica) } \\
\hline & & \multirow{3}{*}{ regimen alterius } & subditi (yconomica) \\
\hline & & & subditi et propinqui \\
\hline & & & \\
\hline
\end{tabular}

De ese modo, ambos textos logran superar la ausencia de sistematicidad interna de la filosofía práctica, caracteristica de la mayoría de las divisiones philosophiae de la primera mitad del siglo. Gracias a esa superación, ambos textos preparan el camino hacia la comprensión de las diferencias entre vir bonus y bonus civis 
que ya eran conocidas en Occidente a través de la ética aristotélica ${ }^{35}$ y que poco despues serían ratificadas y mejor conocidas a través de la Politica. ${ }^{36}$

Tambien Alberto, en su solutio a la segunda pregunta, fundamenta la ética social en un principio que es esencialmente diferente del principio de la ética individual. Alberto llama a este principio homo secundum se en la ética individual y homo in comparatione ad alterum en la ética social. ${ }^{37}$ Solo a partir de este último principio la ética social puede ramificarse en oeconomica y politica. Tambien para Alberto ese principio actúa como principio mediador entre disciplina moralis por una parte y oeconomica y politica por la otra. Actuando como mediador, ese principio posibilita el tránsito desde la disciplina moralis - según la cual homo dupliciter considerari potest - hacia la oeconomica y la politica.

En el esquema de la página anterior pueden percibirse las similitudes entre las estructuras argumentativas de Alberto, del plan de estudios de Barcelona y del escrito Ut ait Tullius. Esas similitudes han sido resaltadas en el esquema correspondiente a cada texto colocándolas en un recuadro de trazo grueso. En ellas encontramos un primer intento de lectura filosófica de la tripartición de la philosophia moralis en ethica, oeconomica y politica y de su fundamentación en principios teóricos que transforman la tripartición asistemática en una bipartición sistemática. Por otra parte, de este esquema surgen además las diferencias que existen entre la asistematicidad de las triparticiones propias de la tradición de las divisiones philosophiae (§ 3) y el intento de los dos textos examinados cuya argumentación retoma Alberto.

\section{5- Conclusión}

A la luz de los elementos precedentes podemos efectuar un balance del proceder de Alberto y de su relevancia en relación con la historia del desarrollo de la ética medieval.

Alberto percibe una evidente insuficiencia en el tratamiento que hace la tradición tanto de las diferencias entre las partes de la philosophia moralis como de su unidad.

En efecto, cuando se ocupa de las diferencias entre ethica, oeconomica y politica dentro del esquema de la philosophia moralis, la tradición fundamenta la distinción entre esas partes de modo tal que resulta imposible reconducirlas a una unidad. Así Alberto verifica que, como consecuencia de dicha insuficiencia, la philosophia moralis pierde la unidad de sus partes.

Alberto verifica además que, a pesar de fundamentar de tal modo la distinción entre las partes que resulta imposible encontrar un vínculo común a ellas, la tradición intenta fundamentar la unidad de la philosophia moralis y que, en ese intento, ella vuelve a errar la elección de los̀ argumentos para fundamentar dicha unidad.

Alberto reacciona frente a ambas actitudes de la tradición. Así, cuando ésta fundamenta la pérdida de la unidad de la philosophia moralis con su consecuente

Cr. Super Ethica, 3a. 
dispersión en sus partes acentuando las diferencias entre éstas, Alberto corrige a la tradición señalándole los fundamentos de la unidad de la philosophia moralis. Pero al mismo tiempo, cuando la tradición pretende fundamentar la unidad de la philosophia moralis con argumentos insuficientes, Alberto recurre a la diferencia entr، ética individual y ética social para señalar dónde están las diferencias.

Pero Alberto no solamente impugna de modo muy consecuente a la tradición, tanto cuando ésta desconoce la unidad de la philosophia moralis como cuando fundamenta su unidad sobre falsos argumentos. De inmediato el mismo Alberto protagoniza el momento positivo de su crítica ocupándose de suministrar argumentos que corrijan los errores que critica. La respuesta de Alberto se empeña en encontrar una solución que, al mismo tiempo que conserve la unidad de la ética, sepa señalar las diferencias entre las partes. La respuesta de Alberto transforma el Prologus al Super Ethica en una suerte de reclamo de una nueva época en favor de la fundamentación científica de la filosofía práctica. Es verdad que en este Prologus se trata aún solo de un primer comienzo que apunta a recuperar los fundamentos científicos tanto de la unidad de las partes de la philosophia moralis como y de las diferencias entre esas partes. De todos modos, este nuevo comienzo introduce a la ética en un nuevo período de su desarrollo, pues él inaugura la búsqueda programática de una nueva y sistemática concepción del esquema tripartito de la philosophia moralis.

Pero el procedimiento de Alberto no se resuelve en su intento de superar la tradición. Mientras lo hace, Alberto menciona argumentos, los critica y luego utiliza otros argumentos cuyo origen histórico es el mismo que el origen de los argumentos que primero había criticado. En la medida en que todos los argumentos a que Alberto recurre - tanto en favor como en contra de la unidad de la ética - provienen del mismo medio intelectual, el procedimiento de Alberto en el Prologus se revela como una asunción de la tradición con el objetivo de someterla a una crítica constructiva y positiva desde dentro mismo de esa tradición utilizando para ello argumentos que encuentra en esa misma tradición. En la medida en que Alberto en parte asume la tradición y en parte la critica, abre a la ética medieval un tránsito no traumático hacia un nuevo período de su desarrollo. De ese modo, el Prologus se muestra como una suerte de punto de inflexión en el que confluye la tradición y a partir del cual ella posibilita la apertura de un nuevo comienzo.

En relación con el problema de la transmisión de las fuentes que confluyen en el pensamiento de Alberto es importante tener en cuenta que aunque el Doctor Universalis identifica las fuentes que menciona con Eustratio, no se trata de una tradición griega, sino que es la tradición latina de las divisiones philosophiae la que mueve a Alberto a criticar la concepción de la ética que recibe de la tradición y que es esa misma tradición latina la que le ofrece argumentos para superar la misma tradición que critica. De allí que resulte de primera importancia tener en cuenta la función desempeñada por la tradición de los artistas en la construcción teórica de los fundamentos de la ética antes de que ingresaran en Occidente todos los escritos éticos y políticos de Aristóteles. 
En un trabajo anterior ${ }^{\circ}$ he analizado esta tradición latina de los maestros de artes con el objetivo de determinar históricamente su contribución como presupuesto y antecedente de la recepción de la Politica de Aristóteles. La interpretación de los pasajes concernientes a la philosophia moralis de las divisiones philosophiae es de fundamental importancia para reconstruir el fenómeno de recepción de la Politica de Aristóteles en Occidente; en efecto, esos pasajes no solamente contienen una implícita concepción acerca de cada parte de la philosophia moralis, sino además una suerte de precomprensión entre las relaciones entre esas partes. Tanto la concepción acerca de las partes como la precomprensión de las relaciones entre ellas constituyen un patrimonio intelectual que con toda verosimilitud debe haber actuado como fundamento de la recepción de la Politica, ya que las divisiones philosophiae hablaron sobre la politica y sobre sus relaciones con las otras partes de la philosophia moralis ya desde antes de que la Politica fuera conocida en Occidente.

Con todo, debe quedar fuera de toda duda que fue recién el ingreso de la Politica en Occidente el fenómeno que contribuyó al conocimiento integral de la philosophia practica y a la determinación de las relaciones entre sus partes. Antes de que ese contenido y esas relaciones pudieran ser definidos con exactitud, las triparticiones de la philosophia practica contenidas en las divisiones philosophiae desempeñaron una función importante como marco de recepción. El desarrollo histórico y filosófico de las concepciones acerca de la ética contenidas en las diferentes triparticiones provenientes del ámbito de los maestros de artes, constituye el preludio a la recepción de los escritos ético-políticos de Aristóteles. El análisis que hemos llevado a cabo de la posición de Alberto frente a las distintas triparticiones de la philosophia moralis ha tenido como objetivo esclarecer un momento de ese desarrollo para incorporarlo a lo que ya conocemos de la historia de la filosofía medieval. a cargo de P. Walter y R. Imbach), Brepols, Tumhout, 1995 (en prensa). 